\title{
An adaptive tone injection for OFDIM PAPR reduction
}

\author{
Mochan Yang and Yoan Shin ${ }^{\text {a) }}$ \\ School of Electronic Engineering, Soongsil University, Seoul 156-743, Korea \\ a)yashin@ssu.ac.kr (corresponding author)
}

\begin{abstract}
We propose an ATI (Adaptive Tone Injection) scheme based on clipping noises for PAPR (Peak-to-Average Power Ratio) reduction of OFDM (Orthogonal Frequency Division Multiplexing) signals. The proposed scheme is composed of three steps: clipping, tone selection, and TI procedures. In the first step, the peak samples in the inverse fast Fourier transform outputs are scaled down by the clipping. In the second step, two tone positions where the powers of the real and imaginary parts of the clipping noises are the maximum, are selected. Finally, the generic TI procedure is performed for the selected tones. Simulation results show that the proposed scheme significantly reduces the complexity of the original TI, while maintaining the PAPR reduction performance.
\end{abstract}

Keywords: orthogonal frequency division multiplexing, peak-to-average power ratio, tone injection, clipping noise

Classification: Wireless communication hardware

\section{References}

[1] L. Hanzo, M. Munster, B. J. Choi, and T. Keller, OFDM and MC-CDMA for Broadband Multi-user Communications, WLANs and Broadcasting, John Wiley \& Sons, 2003.

[2] X. Li and L. J. Cimini, "Effects of clipping and filtering on the performance of OFDM," IEEE Commun. Lett., vol. 2, no. 5, pp. 131-133, May 1998.

[3] R. W. Bauml, R. F. H. Fischer, and J. B. Huber, "Reducing the peakto-average power ratio of multicarrier modulation by selective mapping," IEE Electr. Lett., vol. 32, no. 22, pp. 2056-2057, Oct. 1996.

[4] L. Cimini and N. Sollenberger, "Peak-to-average power ratio reduction of OFDM signal using partial transmit sequences," IEEE Commun. Lett., vol. 4, no. 3, pp. 86-88, March 2000.

[5] J. Tellado, Peak to Average Power Reduction for Multicarrier Modulation, Ph. D. dissertation, Stanford Univ., USA, Stanford, 2000.

[6] B. S. Krongold and D. L. Jones, "An active-set approach for OFDM PAR reduction via tone reservation," IEEE Trans. Signal Process, vol. 52, no. 2, pp. 495-509, Feb. 2004.

[7] C. Tellambura, "Computation of the continuous-time PAR of an OFDM signal with BPSK subcarriers," IEEE Commun. Lett., vol. 5, no. 5, pp. 185-187, May 2001. 


\section{Introduction}

In the transmitters of most wireless communication systems including the OFDM (Orthogonal Frequency Division Multiplexing), HPAs (High Power Amplifiers) are utilized to amplify the transmitted signal power. However, variation of transmitted OFDM signal amplitudes is very wide with large PAPR (Peak-to-Average Power Ratio), which requires expensive HPAs with very good linearity. In addition, large PAPR also demands ADCs (Analogto-Digital Converters) with large dynamic range [1].

For these reasons, many approaches such as clipping, SLM (SeLected Mapping), PTS (Partial Transmit Sequence), ACE (Active Constellation Extension), and TI (Tone Injection) have been proposed to reduce the PAPR of the OFDM signals $[2,3,4,5,6]$. Among these schemes, the TI injects some tones into an OFDM signal by adding constant value [5]. This scheme has the advantage that the BER (Bit Error Rate) will not increase and the only addition to the standard receiver is a modulo operation after the FFT (Fast Fourier Transform). However, a large amount of IFFT (Inverse FFT) and PAPR calculation is required in proportion to the possible combinations within the equivalent constellation set.

We propose in this paper an efficient ATI (Adaptive TI) scheme based on clipping noises to significantly reduce the computational complexity of the conventional TI, while maintaining the PAPR reduction performance.

\section{Conventional tone injection scheme}

The conventional TI reduces the PAPR with no bandwidth loss through duplication of signal constellation points within the equivalent set that will have the same information content. If the duplicated signals are spaced by $D=d \sqrt{M}$ where $M$ is the constellation size and $d$ is the minimum distance between signal points, the BER will not increase and the only addition to the standard receiver is the modulo- $D$ operation after the FFT [5].

The extended QAM (Quadrature Amplitude Modulation) constellation after adding a peak-reduction signal $C_{k}$ is defined by (1), where $X_{k}$ is the symbol of the $k$-th subcarrier (or tone) of the OFDM signals. Note that $\bar{X}_{k}$ in (1) can be easily decoded at the receiver by performing the modulo- $D$ operation.

$$
\bar{X}_{k}=X_{k}+C_{k}=X_{k}+a_{k} D+j b_{k} D, \quad(k=0, \cdots, N-1)
$$

Here, $N$ is the number of sub-carriers, and $a_{k}, b_{k} \in\{+1,-1\}$ denote real and imaginary direction components decided by the TI, respectively.

The transmit signal after the IFFT is then obtained as

$$
\bar{x}_{n}=x_{n}+c_{n}=\frac{1}{\sqrt{N}} \sum_{k=0}^{N-1}\left(X_{k}+a_{k} D+j b_{k} D\right) e^{j \frac{2 \pi k n}{Q N}}, \quad(n=0, \cdots, Q N-1)
$$

In order to include the correct peak samples, the continuous-time OFDM signal is generally sampled at every $T_{s} / Q$ (sec) for $Q$-times oversampling, where $T_{s}$ (sec) is the QAM symbol duration. This can be equivalently achieved by 
using $Q N$-point IFFT after padding $(Q-1) N$ zeros to the original data block of length $N$, as in (2) [7]. We consider $Q=4$-times oversampling throughout the paper.

In order to choose one point within the equivalent set points to minimize the PAPR, an optimal algorithm for the TI requires exponential complexity as in (3) to consider all the possible combinations, which is not practically feasible at all.

$$
\left(\begin{array}{c}
N \\
K
\end{array}\right) S^{K}=\frac{N(N-1) \cdots(N-K+1)}{K !} S^{K} \approx \frac{N^{K}}{K !} S^{K}
$$

where $K(\ll N)$ is the number of injected tones and $S$ is the number of equivalent set points for the injected tones.

\section{Proposed ATI based on clipping noises}

We propose an efficient ATI scheme based on clipping noises to reduce the complexity of the conventional optimal TI. The proposed ATI scheme is composed of three steps: clipping, tone selection, and TI procedures. In practice, these three steps can be adaptively performed for each OFDM symbol.

\subsection{Step 1: Clipping}

In this step, the oversampled input signals $x_{n}(n=0, \cdots, Q N-1)$ are clipped to the maximum amplitude $A_{\max }$ as in (4), while the phase component is maintained as in (5). $A_{\max }$ is determined based on the required clipping ratio $\gamma$ of the system, as defined in (6) [2].

$$
\begin{gathered}
\hat{x}_{n}= \begin{cases}x_{n}, & \text { if }\left|x_{n}\right| \leq A_{\max } \\
A_{\max } e^{j \psi_{n}}, & \text { if }\left|x_{n}\right|>A_{\max }\end{cases} \\
\psi_{n}=\arg \left[x_{n}\right] \\
\gamma=\frac{A_{\max }}{\sqrt{\rho_{s}}}
\end{gathered}
$$

where $\rho_{s}$ is the average power of the input signals. The PAPR is calculated for the IFFT outputs $\mathbf{x}=\operatorname{IFFT}\{\mathbf{X}\}=\left[x_{0}, \cdots, x_{Q N-1}\right]$ of the original input sequence $\mathbf{X}$, and this result is stored for the final TI procedure.

\subsection{Step 2: Tone selection using clipping noises}

In this step, the clipped signals $\hat{x}_{n}$ obtained from Step 1 are used to select the tones which will largely reduce the PAPR.

As the first procedure of this step, the clipping noise is obtained by $x_{c l i p, n}=x_{n}-\hat{x}_{n}(n=0, \cdots, Q N-1)$. Then, the FFT outputs $X_{c l i p, k}(k=$ $0, \cdots, Q N-1)$ of $x_{c l i p, n}$ are obtained by (7).

$$
X_{c l i p, k}=\sum_{s=0}^{N_{0}} x_{c l i p, n_{s}} e^{-j 2 \pi n_{s} k / Q N}, \quad(k=0, \cdots, Q N-1)
$$

Here, $N_{0}$ is the total number of clipping noise samples and $n_{s} \in\left\{n_{0}, \cdots\right.$, $\left.n_{N_{0}-1}\right\}$ is the sub-carrier (or tone) index where the clipping noise sample exists. 
As the next procedure, the sub-carrier positions having the maximum power for real and imaginary parts are obtained by (8) and (9), respectively.

$$
\begin{aligned}
k_{\text {real }}^{*} & =\underset{k \in\{0, \cdots, N-1\}}{\arg \max }\left|\operatorname{Re}\left(X_{k}\right)-\operatorname{Re}\left(X_{\text {clip }, k}\right)\right| \\
k_{\text {imag }}^{*} & =\underset{k \in\{0, \cdots, N-1\}}{\arg \max }\left|\operatorname{Im}\left(X_{k}\right)-\operatorname{Im}\left(X_{\text {clip }, k}\right)\right|
\end{aligned}
$$

Note that $k_{\text {real }}^{*}, k_{\text {imag }}^{*}$ can be regarded as the main contributors generating the peak signal samples, since clipping noise powers in these two tones are larger than in the other tones.

As the final procedure, since the clipping noise signal is a vector, we can obtain direction components $\left(a_{k_{\text {real }}^{*}}, b_{k_{\text {imag }}^{*}}\right)$ from (10).

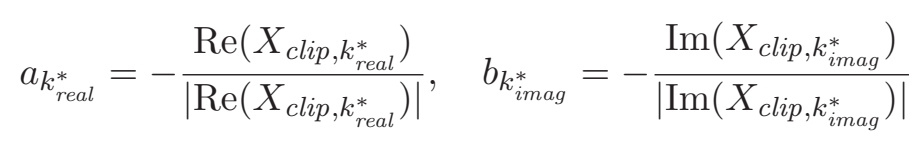

\subsection{Step 3: Generic tone injection}

In this step, we generate the new time-domain signal $\bar{x}_{n}$ as in (11) by using direction components $\left(a_{k_{\text {real }}^{*}}, b_{k_{\text {imag }}^{*}}\right)$ and sub-carrier positions $\left(k_{\text {real }}^{*}, k_{\text {imag }}^{*}\right)$ with maximum clipping noise powers, which are obtained from Step 2.

$$
\bar{x}_{n}=x_{n}+\frac{D}{\sqrt{N}}\left(a_{k_{\text {real }}^{*}} e^{j \frac{2 \pi k_{\text {real }}^{*} n^{n}}{Q N}}+b_{k_{\text {imag }}^{*}} e^{j \frac{2 \pi k_{\text {imag }}^{*}}{Q N}}\right), \quad(n=0, \cdots, Q N-1)
$$

Finally, the signal with the lowest PAPR is selected by comparing the PAPR of the original signal and that of the tone injected signal. Note that $\bar{x}_{n}$ in (11) exhibits low computational complexity because it does not require repeated $Q N$-point IFFT operations.

\section{Simulation results}

To verify the PAPR performance of the proposed ATI, we considered two combinations of $D, N$ and modulation as ( $D=4, N=64$, QPSK) and ( $D=8, N=64,16$-QAM). Signal samples for the transmitted OFDM symbols were oversampled $Q=4$-times to correctly include the peak signal samples [7].

Figure 1 compares PAPR reduction performance in terms of CCDFs (Complementary Cumulative Distribution Functions) of the PAPR for the proposed ATI ("Proposed"), the conventional optimal TI ("Optimal") and Tellado's iterative TI with reduced complexity ("Iterative") [5]. Here, the CCDF is the probability "Prob(PAPR $\left.>\mathrm{PAPR}_{t h}\right)$ " that the actual PAPR is larger than a given threshold, $\mathrm{PAPR}_{t h}$. The figure shows that the proposed ATI achieves comparable PAPR reduction performance to the conventional optimal TI with much less complexity. It is also observed that the proposed scheme achieves almost the same performances of the conventional iterative TI with 8 iterations as shown in Figure 1-(a). 


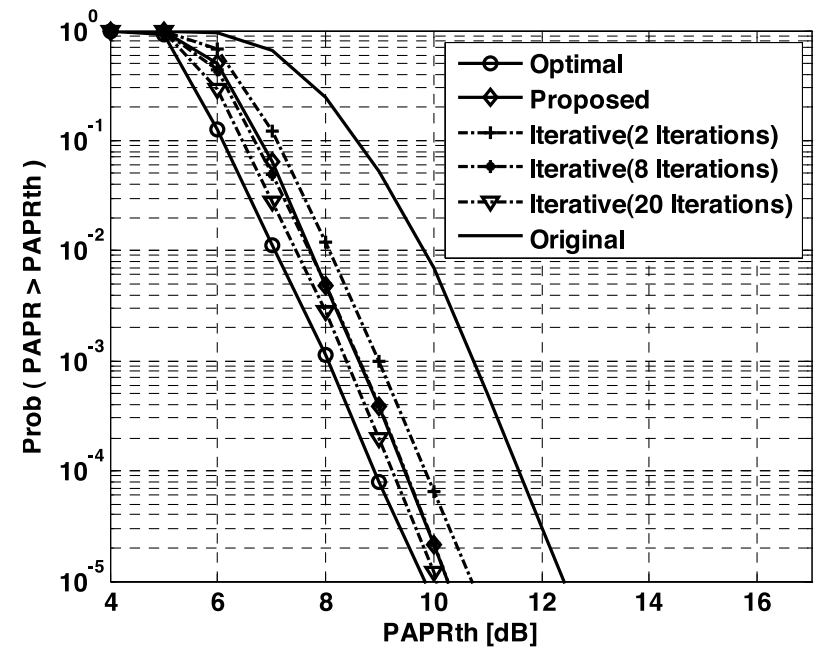

(a) $D=4, N=64$, QPSK

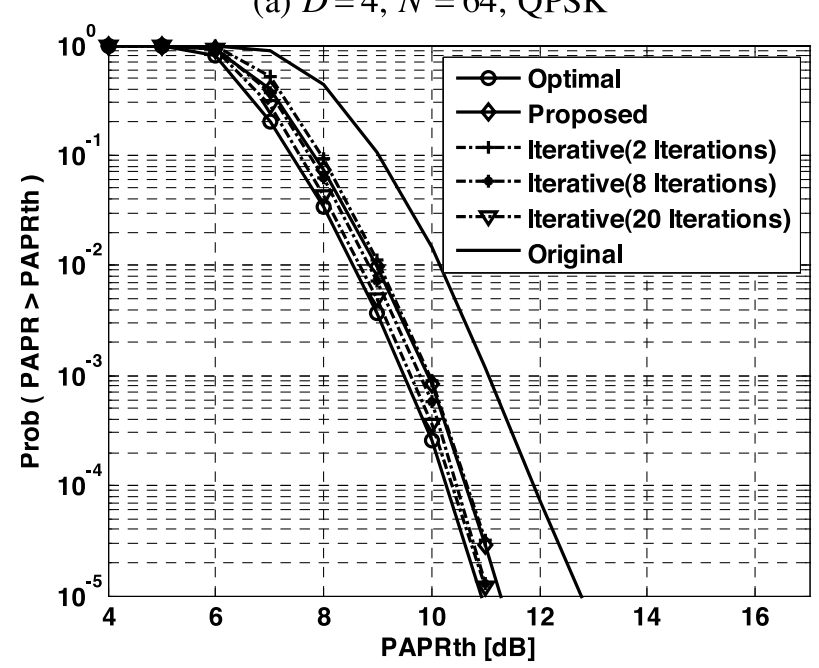

(b) $D=8, N=128,16$-QAM

Fig. 1. Comparison of the PAPR reduction performance among the proposed ATI ("Proposed"), the conventional optimal TI ("Optimal"), and the conventional iterative TI ("Iterative").

Table I. Comparison of the computational complexity $(N=64, S=8, K=2, D=4$, QPSK $)$.

\begin{tabular}{|c|c|c|}
\hline & $\begin{array}{c}\text { \# of } \\
\text { combinations }\end{array}$ & $\begin{array}{c}\text { \# of PAPR } \\
\text { calculations }\end{array}$ \\
\hline \hline Conventional optimal TI & $\left(\begin{array}{c}64 \\
2\end{array}\right) 8^{1}=32,768$ & 32,769 \\
\hline Conventional iterative TI & $\begin{array}{c}8 \cdot 8=64 \\
(8 \text { iterations })\end{array}$ & $\begin{array}{c}(8 \cdot 8)+1=65 \\
(8 \text { iterations })\end{array}$ \\
\hline Proposed ATI & 1 & 2 \\
\hline
\end{tabular}

Tables I and II compare the computational complexity of the three schemes considered. Note that the proposed ATI scheme does not require many possible combinations, in contrast to the conventional schemes. Also, the proposed ATI scheme requires only two PAPR calculations which are 
Table II. Comparison of complexity in terms of the number of additions and multiplications required to obtain the TI symbol of the lowest PAPR $(N=64, S=$ $8, K=2, D=4$, QPSK).

\begin{tabular}{|c|c|c|c|}
\hline & $\begin{array}{c}\text { Conventional } \\
\text { optimal TI }\end{array}$ & $\begin{array}{c}\text { Conventional } \\
\text { iterative TI }\end{array}$ & Proposed ATI \\
\hline \hline Additions & 98,304 & $192(8$ iterations $)$ & 3 \\
\hline Multiplications & 589,824 & $1,152(8$ iterations $)$ & 18 \\
\hline
\end{tabular}

much less than the conventional optimal as well as iterative TI schemes. In addition, the proposed scheme obtains the TI symbol of the lowest PAPR with very small number of actual additions and multiplications.

As shown in the figures and the tables, the proposed ATI scheme achieves comparable PAPR reduction performance of the conventional TI schemes, while significantly reducing the complexity.

\section{Conclusion}

We proposed an efficient ATI scheme based on clipping noises for PAPR reduction of OFDM signals. To reduce the complexity of the conventional TI, the proposed ATI scheme includes clipping, tone selection, and TI steps. After clipping the peak signal samples in the IFFT outputs of the original input sequence, the tone positions having the maximum powers are identified and the direction components are obtained. Then the generic TI procedure is performed for the original input sequence using the direction components. The end result is that the proposed scheme achieves significant reduction of complexity in terms of possible combinations, PAPR calculations, and additions/multiplications relative to the conventional TI, while comparably maintaining the PAPR reduction performance.

\section{Acknowledgments}

This research was partly supported by MKE, Korea under ITRC support program supervised by the NIPA (NIPA-2011-C1090-1111-0005), and by Midcareer Researcher Program through NRF grant funded by MEST, Korea (No. 2011-0016596). 